\title{
Novel Approaches in a Regular Issue
}

The current issue of Acta Baltica Historiae et Philosophiae Scientiarum is, in a way, a regular one. It includes four full-length research articles, two short communications, a book review and a conference overview. Still, there is quite some novelty to be found concerning the content of the papers. To begin with, the approach of Rinat Nugayev to the general relativity theory is obviously an unconventional one. The reviewers expressed different opinions about the article as well. However, after receiving just one clearly negative and several positive, albeit somewhat cautious, reviews, the editor-in-chief decided to publish the paper. After all, one of the main tasks of research papers should be to stimulate discussion and/or bring it to a new level, not just to rephrase thoughts that have been accepted wholeheartedly by the academic community already.

A similar debate developed in the course of reviewing the article by Stanislav Južnič. There was some hesitation on the part of the reviewers representing the Western tradition. However, as the opinion from China was clearly positive, there was no need to consider rejecting this interesting contribution.

The article by Pierdaniele Giarretta and Daniele Chiffi has been written along more conventional methodological lines in philosophy of science but its content can hardly be called traditional in the field. One can also find the second part of a Chinese contribution to logic in the section of research papers. The first part of the article can be found in our previous issue. Thus, Chinese intellectual culture forms one of the main backbones of the current issue.

There is perhaps even more novelty in the section of short communications. To begin with, the papers are actually not that short, but their style does not adhere to that of a typical research article in each and every detail. The contribution by Heldur Sander does not necessarily qualify as belonging to the field of history and philosophy of science. However, it describes very interesting cultural-historical controversies in the Baltic region. These developments are quite typical not only in the territory of today's Republic of Estonia but along almost the whole eastern coast of the Baltic Sea.

John Braidwood's contribution is not quite typical of Acta Baltica either. However, there is really no doubt about the impact of the educational system and its implementation on the development of science anywhere on the face of 
the earth. Needless to say, here, as well as elsewhere throughout the journal, our editorial team does not intervene in the right of any author to decide on the angle of their approach to the topics they employ as well as in expressing their opinion concerning any issue they touch upon in the writings. This will remain the responsibility of the authors themselves.

A short overview of an interesting conference on the heritage of the Russian female scientists concludes the issue.

I wish you all a good time in the company of our journal!

Peeter Mü̈̈rsepp

Editor-in-Chief 